Check for updates

Cite this: RSC Adv., 2019, 9, 24981

\title{
Theoretical investigations of thermoelectric phenomena in binary semiconducting skutterudites
}

Received 23rd May 2019

Accepted 24th June 2019

DOI: $10.1039 /$ c9ra03882e

rsc.li/rsc-advances

\author{
Muhammad Saeed, (D) a Banaras Khan, ${ }^{\text {bcd }}$ Iftikhar Ahmad, ${ }^{\text {bc }}$ Awais Siddique Saleemi,
} Najeebur Rehman, ${ }^{f}$ H. A. Rahnamaye Aliabad (DD ${ }^{g}$ and Sarir Uddin ${ }^{d}$

In this study, we explored the thermoelectric properties of the host thermoelectric materials (TM), namely, binary skutterudites, using a combination of simulations based on density functional theory and post-DFT Boltzmann's semiclassical theory. The calculations were performed close to the Fermi surface for the Seebeck coefficient and other thermoelectric parameters. Our results demonstrated that $\mathrm{CoSb}_{3}$ exhibited the highest Seebeck value at room temperature among all the compounds $\left(\mathrm{CoP}_{3}, \mathrm{CoAs}_{3}\right.$, $\mathrm{CoSb}_{3}, \mathrm{IrP}_{3}, \mathrm{IrAs}_{3}, \mathrm{IrSb}_{3}, \mathrm{RhAs}_{3}$, and $\left.\mathrm{RhSb}_{3}\right)$, which confirmed that this compound is an ideal host material for thermoelectric applications. Furthermore, the calculated electrical conductivity values show that $\mathrm{RhAs}_{3}$ has the largest value of $3.736 \times 10^{5} \Omega^{-1} \mathrm{~m}^{-1}$. However, at high temperatures, the Seebeck values for all of these compounds are almost constant due to the activation of the minority charge carriers.

\section{Introduction}

The demand for energy has caused a remarkable unrest across the globe and climate change due to the combustion of fossil fuels is increasing and creating a drastic impact on the social and economic life of human civilization. ${ }^{1}$ In this context, the conversion of waste heat from various sources to useful electrical energy can play a key role in the energy crisis as well as growing environmental problem. The heating process of buildings, heat from the automobile exhausts as well as industries, all generate a huge amount of waste heat that could be converted to electricity using thermoelectric (TE) generators. Thermoelectric generators are solid devices having no moving parts, make no noise, reliable and scalable, which make them ideal for small amounts of electric power generation. ${ }^{\mathbf{1 , 2}}$ It is estimated that two-third of used energy is lost as waste heat, so there is a need for good TE materials that can be used to convert heat to electrical energy and vice versa. ${ }^{3}$

Certain materials are identified for their high efficiency of energy conversion from heat to electricity with a high TE figure

\footnotetext{
${ }^{a}$ College of Nuclear Science and Engineering, East China University of Technology, Nanchang, 330013, China

${ }^{b}$ Center for Computational Materials Science, University of Malakand, Chakdara, Pakistan.E-mail: banarasphysicist@gmail.com

${ }^{c}$ Department of Physics, University of Malakand, Chakdara, Pakistan

${ }^{d}$ Department of Physical and Numerical Sciences, Qurtuba University of Science \& Information Technology, Peshawar, Pakistan

${ }^{e}$ Key Laboratory of Optoelectronic Devices and Systems of Ministry of Education and Guangdong Province, College of Optoelectronic Engineering, Shenzhen University, Shenzhen 518060, Guangdong, China

${ }^{f}$ Department of Chemistry, University of Malakand, Chakdara, Pakistan

${ }^{g}$ Department of Physics, Hakim Sabzevari University, Sabzevar, Iran
}

of merit above unity. These materials have good electrical conductivity, and ultimately maintain a low thermal conductivity. Their interesting results have attracted considerable attention and research is still in progress to further improve their TE properties. ${ }^{4}$ High-temperature bulk materials, such as skutterudites, clathrates, half-heusler alloys and complex chalcogenides, have been extensively studied for their TE applications. ${ }^{5}$ These materials usually have complex crystal structures and physical properties that are required for a good TE material. For example, the skutterudite-structured compounds and clathrates have cage-like structures that have empty spaces where filler atoms are inserted, which significantly lower the thermal conductivity of these compounds due to the filling atoms' ability to scatter phonons. These materials possess some unusual phenomena, such as heavy fermion superconductivity, an exciton-mediated superconducting state and Weyl fermions. ${ }^{6}$

Skutterudites have attracted significant attention as high temperature TE materials due to their high figure of merit $(z T)$ in the temperature range from $300{ }^{\circ} \mathrm{C}$ to $550{ }^{\circ} \mathrm{C} .^{7}$ The experimental observations show that skutterudite semiconductors possess attractive transport properties and have good potential for achieving $z T$ values. For p-type skutterudites, low hole effective mass, high carrier mobility, low electrical resistivity and moderate Seebeck coefficient were obtained; however, the larger electron effective mass and Seebeck coefficients make the n-type skutterudites more attractive candidates for TE applications. ${ }^{8}$ The Seebeck coefficient values of all skutterudites are high and therefore can be used as thermoelectric materials. ${ }^{9}$ However, $\mathrm{CoSb}_{3}$ has attracted enormous attention for its conversion applications of waste heat to electricity due to its reasonable band gap, high carrier mobility and the fact that it is 
composed of inexpensive and environmentally friendly elements as compared to other skutterudite materials. The thermal conductivity of pure $\mathrm{CoSb}_{3}$ is, however, very high, which leads to a low $z T$ and poor conversion efficiency for TE applications. ${ }^{\mathbf{1 0}}$

The thermoelectric properties of the binary skutterudite have been studied extensively via experiments. However, some theoretical studies are available on the filled ones, but no detailed theoretical studies are available on the comparison of the binary skutterudites. The motivation of the present study is an attempt to understand the theory of TE properties of the binary skutterudite compounds.

\section{Theory and calculations}

The Boltzmann theory discusses a variation in the charge carriers' distribution function due to external fields, lattice phonon scattering, or various kinds of defect scattering. For the situation of an electric and magnetic fields and a thermal gradient, the electric current $j$ can be written in terms of the tensor form as

$$
j_{i}=\sigma_{i j} E_{j}+\sigma_{i j} k E_{j} B k+\nu_{i j} \nabla_{j} T
$$

However, the complexity of various carrier scattering mechanisms causes difficulty in obtaining an exact solution of the Boltzmann transport equation. ${ }^{\mathbf{1 1} 12}$ Mathematically, the relaxation time is given by

$$
\frac{\delta f}{\delta t}\left(\frac{\delta f}{\delta t}\right)_{s}=-\frac{f-f_{0}}{\tau}
$$

where $f_{0}$ and $f$ are the equilibrium and perturbed carrier distribution functions, respectively, and $\tau$ is the relaxation time. eqn (2) describes the process of restoring $f_{0}$ from $f$ via various scattering processes. The net effect is to restore $f$ to $f_{0}$ exponentially with a relaxation time $\tau$. Basically, $\tau$ is very complicated; however, in this study, $\tau$ has been approximated. ${ }^{\mathbf{1 2 - 2 0}}$ Using this approximation, the thermoelectric parameters in the tensor form for a material can be written as

$$
\begin{gathered}
\sigma_{\alpha \beta}(T, \mu)=\frac{1}{\Omega} \int \bar{\sigma}_{\alpha \beta}(\varepsilon)\left[-\frac{\sigma f_{0}(T, \alpha, \mu)}{\delta s}\right] \mathrm{d} \varepsilon \\
S_{\alpha \beta}(T, \mu)=\frac{1}{e T \Omega \sigma_{\alpha \beta}(T, \mu)} \int \bar{\sigma}_{\alpha \beta}(\varepsilon)(\varepsilon-\mu)\left[-\frac{\sigma f_{0}(T, \alpha, \mu)}{\delta s}\right] \mathrm{d} \varepsilon
\end{gathered}
$$

where $\alpha$ and $\beta$ are the tensor indices, and $\Omega, \mu$ and $f_{0}$ are the volume of the unit cell, Fermi level of carriers, and the carrier Fermi-Dirac distribution function, respectively, and $e$ is the electronic charge. The transport distribution function tensor given in $\sigma$ and $S$ is defined as:

$$
\bar{\sigma}_{\alpha \beta}(\varepsilon)=\frac{e}{N} \sum_{i, k} \tau \nu_{\alpha}(i, k) \nu_{\beta}(i, k) \frac{\delta\left(\delta-\delta_{i, k}\right)}{\delta_{s}}
$$

where $k$ and $i$ are the wave vector and band index, respectively, and $N$ represents the number of $k$ points. In the three dimensional tensor, $\nu \alpha(i, k)(\alpha=x, y, z)$ is the $\alpha$-th component of the group velocity $\nu(i, k)$ of carriers, where it can be derived directly from the band structure by

$$
\nu(i, k)=\frac{1}{h} \nabla_{k, s_{i, k}}
$$

The so-called band-crossing ${ }^{17}$ may introduce problems in calculating $v(i, k)$ from band structures. In these calculations, the band structures of these binary skutterudites were calculated in comparison to the optical band gaps obtained by experimental measurements, as given in Fig. 1. The electronic band structure calculations were performed using the improved Tran-Blaha modified Becke-Johnson potential (improved TB$\mathrm{mBJ}){ }^{21}$ This newly developed technique yields very accurate electronic band structures and band gaps for various types of semiconductors and insulators in comparison to the wellknown Green's function method (GW) and Tran-Blaha modified Becke-Johnson (TB-mBJ) technique. Furthermore, the details about the electronic structure of the binary skutterudites and improved TB-mBJ can be found in our previous study. ${ }^{22}$ To calculate the thermoelectric parameters, a dense $k$ mesh of $56000 \mathrm{k}$ points was used in this study as per the method proposed by Madsen and Singh. ${ }^{17}$ By using eqn (2)-(5), the Seebeck coefficient and electrical conductivity of a compound can be estimated based on its band structure. The carrier relaxation time $t$ is taken to be a constant with the value approximated to $10-13 \mathrm{s.}^{23}$ The electrical conductivity and power factor were calculated with respect to $\tau$; the Seebeck coefficient is independent of $\tau$. This approach has been employed successfully in evaluating the electrical transport properties of thermoelectric compounds. ${ }^{\mathbf{1 4 - 1 8 , 2 4}}$ Following similar procedures, we have interfaced this methodology with the Wien $2 \mathrm{k}$ code. ${ }^{25}$

\section{Results and discussions}

\section{Seebeck coefficient}

Thermoelectric phenomenon in materials can be quantified by different parameters, one of which is the Seebeck coefficient, which has a unique importance because of its voltage capability for a given temperature gradient. A high Seebeck coefficient value usually leads to a good thermoelectric material. The Seebeck coefficient is a tool that provides a sensitive test of the

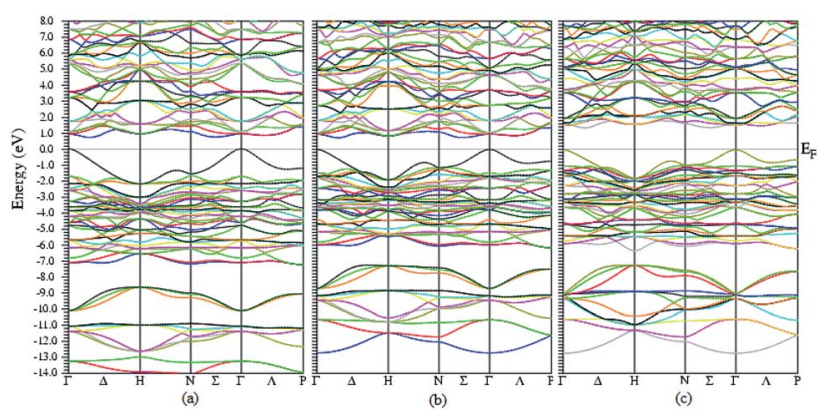

Fig. 1 Calculated band structures of (a) $\mathrm{RhAs}_{3}$, (b) RhSb 3 and. (c) $\mathrm{IrSb}_{3}$ by the improved TB-mBJ. 
electronic structure in the vicinity of the Fermi energy. A different electronic structure results in different values for the Seebeck coefficient. The temperature dependence of the Seebeck coefficients $S$ for the semiconducting binary skutterudites is shown in Fig. 2. Large values, with magnitudes on the order of $\mu \mathrm{V} \mathrm{K}^{-1}$, were observed in all samples. The calculated values of the Seebeck coefficients at room temperature were in close agreement with experimental values as listed in Table 1. In Fig. 2, the Seebeck coefficient increased with the increase in temperature up to $700 \mathrm{~K}$ and tended to flatten at higher temperatures. This is due to an increase in the number of thermally excited minority carriers, which tend to decrease the magnitude of the Seebeck coefficient. For all binary skutterudites an increase in temperature increases the energy per charge and ultimately increases the voltage (i.e., the Seebeck coefficient), and also exhibits positive $S$ values, showing that the majority carriers are holes. In these calculations, the maximum observed value of the Seebeck coefficient was for $\mathrm{CoSb}_{3}$, which was $215 \mu \mathrm{V} \mathrm{K}^{-1}$ at room temperature. The Seebeck coefficient at $50 \mathrm{~K}$ for $\mathrm{CoP}_{3}$ in our calculation was $0.8782 \mu \mathrm{V} \mathrm{K}{ }^{-1}$. As the temperature rose, the calculated curve for $\mathrm{CoP}_{3}$ linearly increased with temperature to around $44 \mu \mathrm{V} \mathrm{K}{ }^{-1}$ in about $300 \mathrm{~K}$ and this increase was continuous up to $800 \mathrm{~K}$, at which point the Seebeck coefficient became $103 \mu \mathrm{V} \mathrm{K}^{-1}$. The Seebeck curve of $\mathrm{CoAs}_{3}$ first at $50 \mathrm{~K}$ was $41 \mu \mathrm{V} \mathrm{K}^{-1}$, and then it increased to around $145 \mu \mathrm{V} \mathrm{K}^{-1}$ at about $300 \mathrm{~K}$, and finally increased to about $189 \mu \mathrm{V} \mathrm{K}^{-1}$ at $800 \mathrm{~K}$. Our calculated curves of $\mathrm{CoSb}_{3}$ first increased to around $215 \mu \mathrm{V} \mathrm{K}^{-1}$ at about $300 \mathrm{~K}$, and then this increase reached about $279 \mu \mathrm{V} \mathrm{K}^{-1}$ at $800 \mathrm{~K}$. The calculated curve of $\mathrm{IrP}_{3}$ first abruptly increased to a value of around $199 \mu \mathrm{V}$ $\mathrm{K}^{-1}$ at about $300 \mathrm{~K}$, and then showed a tendency of small increases and reached about $224 \mu \mathrm{V} \mathrm{K}^{-1}$ at about $800 \mathrm{~K}$. Our calculated curves of $\mathrm{IrAs}_{3}$ estimated the value of the Seebeck coefficient to be about $44.6 \mu \mathrm{V} \mathrm{K}^{-1}$ at about $50 \mathrm{~K}$, and then increased up to a value of $200 \mu \mathrm{V} \mathrm{K}^{-1}$ at $800 \mathrm{~K}$. However, at room temperature, the calculated Seebeck coefficient value was 148 $\mu \mathrm{V} \mathrm{K}^{-1}$. The trend followed by the Seebeck coefficient for $\mathrm{IrSb}_{3}$ was the same as that for $\operatorname{IrP}_{3}$ and $\mathrm{IrAs}_{3}$, as is clear from Fig. 2 . The values of the Seebeck coefficients at the temperatures 50,

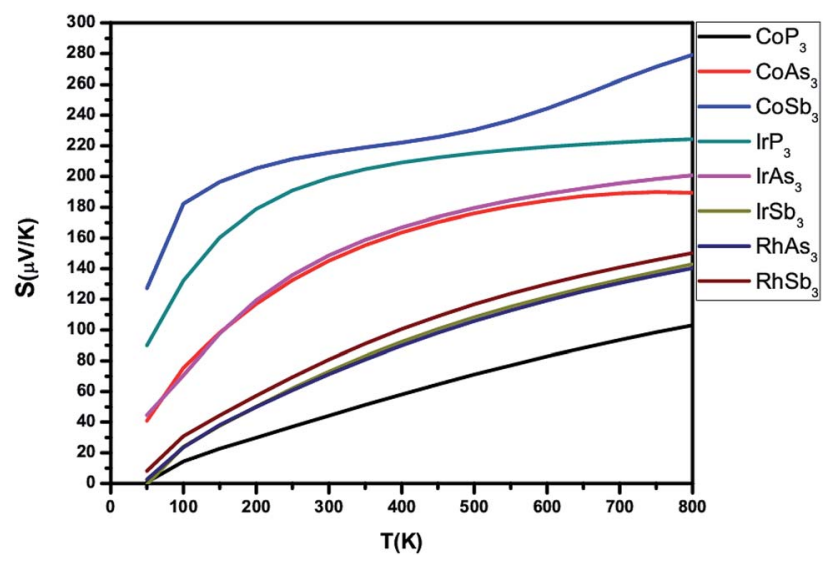

Fig. 2 Seebeck coefficient versus the temperature of the binary skutterudites.
$300,800 \mathrm{~K}$ are $18.086 \mu \mathrm{V} \mathrm{K}{ }^{-1}, 70.0 \mu \mathrm{V} \mathrm{K}^{-1}$ and $139.4565 \mu \mathrm{V} \mathrm{K}^{-1}$, respectively. The Seebeck coefficient values for $\mathrm{RhAs}_{3}$ showed a regular increasing trend with temperature and the calculated values were $2.6473 \mu \mathrm{V} \mathrm{K}^{-1}, 71.23732 \mu \mathrm{V} \mathrm{K}^{-1}$ and $140.29174 \mu \mathrm{V}$ $\mathrm{K}^{-1}$ at 50, 300 and $800 \mathrm{~K}$, respectively. Finally, the results obtained for $\mathrm{RhSb}_{3}$ at the temperatures 50, 300 and $800 \mathrm{~K}$ were $8.15587 \mu \mathrm{V} \mathrm{K} \mathrm{K}^{-1}, 80.79364 \mu \mathrm{V} \mathrm{K} \mathrm{K}^{-1}$ and $150.18459 \mu \mathrm{V} \mathrm{K} \mathrm{K}^{-1}$, respectively.

\section{Electrical conductivity}

The electrical conductivity is the passage of the charge carriers through a material. In a semiconductor, both the electrons and holes are responsible for the electrical conductivity. In these compounds, the electrical conductivity almost linearly increased with temperature, which was due to the increase in the number of carriers and potential, as shown in Fig. 3. The electrical conductivity of these compounds was of the order $10^{4}$ $\Omega^{-1} \mathrm{~m}^{-1}$.

The calculated and experimental values of the electrical conductivity at room temperature are listed in Table 1 and our comparative study shows that the calculated values of the electrical conductivity agreed with the experimental values. The maximum value of the electrical conductivity in the binary skutterudites was obtained for $\mathrm{RhAs}_{3}$ for all calculated temperatures in the range from 50 to $800 \mathrm{~K}$. The calculated electrical conductivity of $\mathrm{CoP}_{3}$ at $50 \mathrm{~K}$ was $0.6645 \times 10^{4} \Omega^{-1}$ $\mathrm{m}^{-1}$, and then linearly increased to $2.06379 \times 10^{4} \Omega^{-1} \mathrm{~m}^{-1}$ at room temperature. Subsequently, the electrical conductivity increased gradually and reached a peak value of $9.9643 \times 10^{4}$ $\Omega^{-1} \mathrm{~m}^{-1}$ at $800 \mathrm{~K}$. For $\mathrm{CoAs}_{3}$ at $50 \mathrm{~K}$, the electrical conductivity was $0.001592 \times 10^{4} \Omega^{-1} \mathrm{~m}^{-1}$ and reached $0.32 \times 10^{4} \Omega^{-1} \mathrm{~m}^{-1}$ at $300 \mathrm{~K}$. This increase in the electrical conductivity was continuous up to $4.643 \times 10^{4} \Omega^{-1} \mathrm{~m}^{-1}$ at $800 \mathrm{~K}$. The calculated conductivity for $\mathrm{CoSb}_{3}$ at $50 \mathrm{~K}$ was $0.2385 \times 10^{4} \Omega^{-1} \mathrm{~m}^{-1}$ and increased linearly with temperature to a value of $2.05247 \times 10^{4}$ $\Omega^{-1} \mathrm{~m}^{-1}$ at $300 \mathrm{~K}$. This increase continued until the electrical conductivity reached $9.35315 \times 10^{4} \Omega^{-1} \mathrm{~m}^{-1}$ at $800 \mathrm{~K}$. The obtained results for $\operatorname{IrP}_{3}$ at $50 \mathrm{~K}$ for the electrical conductivity was $1.58836 \times 10^{4} \Omega^{-1} \mathrm{~m}^{-1}$, and the increase in temperature of $\operatorname{IrP}_{3}$ led to an electrical conductivity value of $3.8848 \times 10^{4} \Omega^{-1} \mathrm{~m}^{-1}$ at $300 \mathrm{~K}$ and $11.83157 \times 10^{4} \Omega^{-1} \mathrm{~m}^{-1}$ at $800 \mathrm{~K}$. The electrical conductivity of $\mathrm{IrAs}_{3}$ at $50 \mathrm{~K}$ was $1.4595 \times 10^{4} \Omega^{-1} \mathrm{~m}^{-1}$, and then increased to $3.32611 \times 10^{4} \Omega^{-1} \mathrm{~m}^{-1}$. The increase in temperature caused a further increase in the electrical conductivity to a value of $8.88461 \times 10^{4} \Omega^{-1} \mathrm{~m}^{-1}$ at $800 \mathrm{~K}$. The calculated value of the electrical conductivity for $\mathrm{IrSb}_{3}$ at $50 \mathrm{~K}$ was $2.363589 \times 10^{5} \Omega^{-1} \mathrm{~m}^{-1}$ and became $2.291882 \times 10^{5} \Omega^{-1}$ $\mathrm{m}^{-1}$ at $300 \mathrm{~K}$, which was close to the experimental value at this temperature with the value of $2.33 \times{ }^{5} \Omega^{-1} \mathrm{~m}^{-1}$. A further increase in the temperature increased the electrical conductivity and reached $2.8827 \times 10^{5} \Omega^{-1} \mathrm{~m}^{-1}$ at $800 \mathrm{~K}$. A calculation for the electrical conductivity of $\mathrm{RhAs}_{3}$ gave the value of $3.5667 \times$ $10^{5} \Omega^{-1} \mathrm{~m}^{-1}$ at $50 \mathrm{~K}$, which changed by increasing the temperature and became $3.7361 \times 10^{5} \Omega^{-1} \mathrm{~m}^{-1}$ at a temperature of $300 \mathrm{~K}$. Finally, the curve reached $4.2289 \times 10^{5} \Omega^{-1} \mathrm{~m}^{-1}$ at $800 \mathrm{~K}$. The electrical conductivity values for $\mathrm{RhSb}_{3}$ showed the 
Table 1 Calculated values of the thermoelectric parameters in comparison with experimental values at room temperature

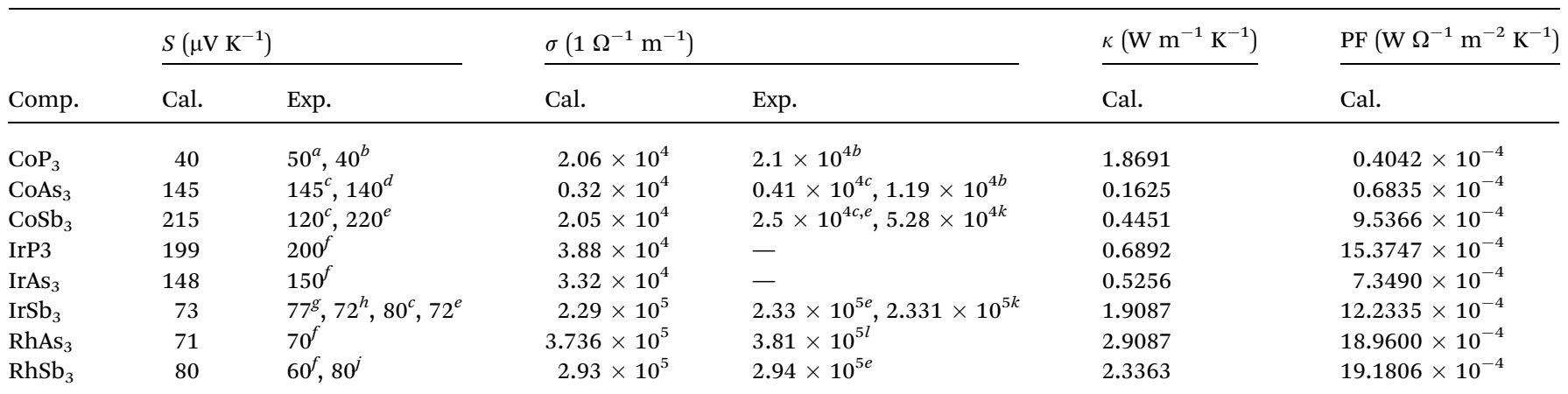

${ }^{a}$ Ref.26. ${ }^{b}$ Ref. 27. ${ }^{c}$ Ref. $28 .{ }^{d}$ Ref. 29. ${ }^{e}$ Ref. $30 .{ }^{f}$ Ref. $31 .{ }^{g}$ Ref. $32 .{ }^{h}$ Ref. $33 .{ }^{i}$ Ref. $34 .{ }^{j}$ Ref. $35 .{ }^{k}$ Ref. $36 .{ }^{l}$ Ref. 37.

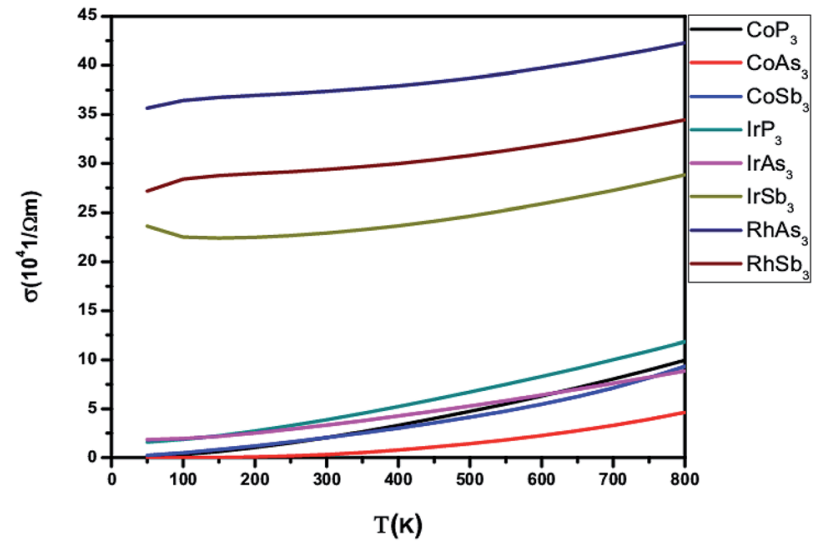

Fig. 3 Electrical conductivity versus the temperature of the binary skutterudites.

same trend and the values at 50, 300 and $800 \mathrm{~K}$ were $2.7174 \times$ $10^{5} \Omega^{-1} \mathrm{~m}^{-1}, 2.9383 \times 10^{5} \Omega^{-1} \mathrm{~m}^{-1}$ and $3.4463 \times 10^{4} \Omega^{-1} \mathrm{~m}^{-1}$, respectively.

\section{Electronic thermal conductivity}

Thermal conductivity is the flow of heat in materials and it arises from both the electronic and lattice parts of the compound, as shown by $k=k e+k l$. However, the BoltzTraP code can only estimate the electronic part of the thermal conductivity $k e$. The electronic thermal conductivity for the semiconducting binary skutterudites is tabulated in Table 1 as well as depicted in Fig. 4. The electronic thermal conductivity variation with temperature was prominent and changed abruptly with temperature. It was found for $\mathrm{CoP}_{3}$ that the electronic thermal conductivity $\mathrm{K}$ at $50 \mathrm{~K}$ was $0.23945 \mathrm{~W} \mathrm{~K}^{-1}$ $\mathrm{m}^{-1}$, which then increased with temperature and became $1.86913 \mathrm{~W} \mathrm{~K}^{-1} \mathrm{~m}^{-1}$ at $300 \mathrm{~K}$. The value was increased to $10.81445 \mathrm{~W} \mathrm{~K}^{-1} \mathrm{~m}^{-1}$ at $800 \mathrm{~K}$. The $\mathrm{CoAs}_{3}$ calculated electronic thermal conductivity at a temperature of $50 \mathrm{~K}$ was $0.1218 \mathrm{~W} \mathrm{~K}^{-1}$ $\mathrm{m}^{-1}$ and was $0.1624 \mathrm{~W} \mathrm{~K}^{-1} \mathrm{~m}^{-1}$ at room temperature $(300 \mathrm{~K})$. With a further increase in the temperature to $800 \mathrm{~K}$, the electronic thermal conductivity then became $5.4099 \mathrm{~W} \mathrm{~K}^{-1} \mathrm{~m}^{-1}$. The $\mathrm{CoSb}_{3}$ electronic thermal conductivity obtained in our calculations at $50 \mathrm{~K}$ was $0.00646 \mathrm{~W} \mathrm{~K}^{-1} \mathrm{~m}^{-1}$. At a temperature of $300 \mathrm{~K}$, the thermal conductivity of $\mathrm{CoSb}_{3}$ became $0.44515 \mathrm{~W} \mathrm{~K}^{-1}$ $\mathrm{m}^{-1}$, and then the electronic thermal conductivity further increased to a value of $9.71322 \mathrm{~W} \mathrm{~K}^{-1} \mathrm{~m}^{-1}$. The calculated values of the electronic thermal conductivity also showed an increase with temperature for $\operatorname{IrP}_{3}$. At the temperatures 50, 300 and $800 \mathrm{~K}$, the thermal conductivity values were 0.02381 , 0.68928 and $6.56185 \mathrm{~W} \mathrm{~K}^{-1} \mathrm{~m}^{-1}$, respectively. The calculations performed for $\mathrm{IrAs}_{3}$ determined that the electronic thermal conductivity at $50 \mathrm{~K}$ was $0.02376 \mathrm{~W} \mathrm{~K}^{-1} \mathrm{~m}^{-1}$ and increased with the increase in temperature, by which the resultant values at 300 and $800 \mathrm{~K}$ were 0.5256 and $4.8096 \mathrm{~W} \mathrm{~K}^{-1} \mathrm{~m}^{-1}$, respectively. The thermal conductivity findings for $\mathrm{IrSb}_{3}$ showed a similar behavior as discussed for the previous skutterudite materials, and showed a continuous increase with temperature. The calculated values at the temperatures 0,300 and $800 \mathrm{~K}$ were $0.29456,2.01718$ and $10.00149 \mathrm{~W} \mathrm{~K}^{-1} \mathrm{~m}^{-1}$, respectively. The $\mathrm{RhAs}_{3}$ material had an electronic thermal conductivity value of $0.44776 \mathrm{~W} \mathrm{~K}^{-1} \mathrm{~m}^{-1}$ at $50 \mathrm{~K}$, and then became $2.90874 \mathrm{~W} \mathrm{~K}^{-1}$ $\mathrm{m}^{-1}$ at $300 \mathrm{~K}$ and finally $11.8569 \mathrm{~W} \mathrm{~K}^{-1} \mathrm{~m}^{-1}$ at $800 \mathrm{~K}$. Our calculations for $\mathrm{RhSb}_{3}$ at $50 \mathrm{~K}$ estimated the electronic thermal conductivity value at $0.03378 \mathrm{~W} \mathrm{~K}^{-1} \mathrm{~m}^{-1}$. Increasing the temperature to $300 \mathrm{~K}$, the electronic thermal conductivity value showed an increase to the value $0.68953 \mathrm{~W} \mathrm{~K}^{-1} \mathrm{~m}^{-1}$. At $800 \mathrm{~K}$, the thermal conductivity became $5.87658 \mathrm{~W} \mathrm{~K}^{-1} \mathrm{~m}^{-1}$.

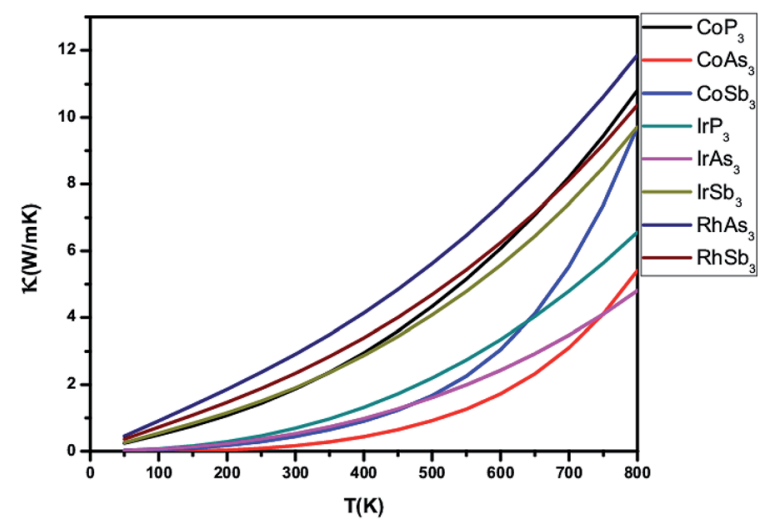

Fig. 4 Variation of electronic thermal conductivity of skutterudites with temperature. 


\section{Power factor}

The power factor is one of the parameters used to measure the thermoelectric efficiency of a compound. Mathematically, it is given as $\mathrm{PF}=S^{2} \sigma$, where $S$ is the Seebeck coefficient and $\sigma$ is the electrical conductivity of the material. The power factor, instead of $S$ or $\sigma$, is a comprehensive parameter for the electrical performance of a compound. The calculated values of the power factor for the binary skutterudite compounds are tabulated in Table 1. The maximum power factor in a skutterudite semiconductor system is for $\mathrm{RhAs}_{3}$, with the peak value of $83.233 \times$ $10^{-4} \mathrm{~W} \Omega^{-1} \mathrm{~m}^{-2} \mathrm{~K}^{-1}$ at $800 \mathrm{~K}$, as shown in Fig. 5. For the compound $\mathrm{CoP}_{3}$, the power factor at $50 \mathrm{~K}$ temperature is very low with a value of $5.1228 \times 10^{-10} \mathrm{~W} \Omega^{-1} \mathrm{~m}^{-2} \mathrm{~K}^{-1}$. With the increase in temperature to $300 \mathrm{~K}$ power factor becomes 0.4042 $\times 10^{-4} \mathrm{~W} \Omega^{-1} \mathrm{~m}^{-2} \mathrm{~K}^{-1}$ and finally at $800 \mathrm{~K}$, the power factor of $\mathrm{CoP}_{3}$ then became $10.5941 \times 10^{-4} \mathrm{~W} \Omega^{-1} \mathrm{~m}^{-2} \mathrm{~K}^{-1}$.

The calculations for $\mathrm{CoAs}_{3}$ at $50 \mathrm{~K}$ gave us a power factor value of $2.66 \times 10^{-11} \mathrm{~W} \Omega^{-1} \mathrm{~m}^{-2} \mathrm{~K}^{-1}$. Then, with an increase in the temperature to $300 \mathrm{~K}$, the power factor increased to a value of $0.68351 \times 10^{-4} \mathrm{~W} \Omega^{-1} \mathrm{~m}^{-2} \mathrm{~K}^{-1}$. Finally, at $800 \mathrm{~K}$, the power factor became $16.6251 \times 10^{-4} \mathrm{~W} \Omega^{-1} \mathrm{~m}^{-2} \mathrm{~K}^{-1}$. The estimated value of the power factor for $\mathrm{CoSb}_{3}$ at $50 \mathrm{~K}$ was $0.3863 \times 10^{-4} \mathrm{~W}$ $\Omega^{-1} \mathrm{~m}^{-2} \mathrm{~K}^{-1}$ and increased with temperature to the values $9.5366 \times 10^{-4} \mathrm{~W} \Omega^{-1} \mathrm{~m}^{-2} \mathrm{~K}^{-1}$ and $72.9146 \times 10^{-4} \mathrm{~W} \Omega^{-1} \mathrm{~m}^{-2}$ $\mathrm{K}^{-1}$ at 300 and $800 \mathrm{~K}$, respectively. The results of the power factor for $\mathrm{IrP}_{3}$ at the temperatures 50,300 and $800 \mathrm{~K}$ are 1.2846 $\times 10^{-4} \mathrm{~W} \Omega^{-1} \mathrm{~m}^{-2} \mathrm{~K}^{-1}, 15.3747 \times 10^{-4} \mathrm{~W} \Omega^{-1} \mathrm{~m}^{-2} \mathrm{~K}^{-1}$ and $59.5952 \times 10^{-4} \mathrm{~W} \Omega^{-1} \mathrm{~m}^{-2} \mathrm{~K}^{-1}$, respectively. The $\mathrm{IrAs}_{3}$ values of the power factor showed an usual increase with temperature, and the values obtained at the temperatures 50,300 and $800 \mathrm{~K}$ are $0.3672 \times 10^{-4} \mathrm{~W} \Omega^{-1} \mathrm{~m}^{-2} \mathrm{~K}^{-1}, 7.3490 \times{ }^{-4} \mathrm{~W} \Omega^{-1} \mathrm{~m}^{-2} \mathrm{~K}^{-1}$ and $35.7767 \times 10^{-4} \mathrm{~W} \Omega^{-1} \mathrm{~m}^{-2} \mathrm{~K}^{-1}$, respectively.

Our calculations for $\mathrm{IrSb}_{3}$ gave a value of $0.0011 \times 10^{-4} \mathrm{~W}$ $\Omega^{-1} \mathrm{~m}^{-2} \mathrm{~K}^{-1}, 12.2335 \times 10^{-4} \mathrm{~W} \Omega^{-1} \mathrm{~m}^{-2} \mathrm{~K}^{-1}$ and $58.8840 \times 10^{-}$ ${ }^{4} \mathrm{~W} \Omega^{-1} \mathrm{~m}^{-2} \mathrm{~K}^{-1}$ for the power factor at 50, 300 and $800 \mathrm{~K}$, respectively. The calculations performed for $\mathrm{RhAs}_{3}$ showed an increase with temperature, and the results obtained at 50, 300 and $800 \mathrm{~K}$ are $0.0250 \times 10^{-4} \mathrm{~W} \Omega^{-1} \mathrm{~m}^{-2} \mathrm{~K}^{-1}, 18.9600 \times 10^{-4} \mathrm{~W}$ $\Omega^{-1} \mathrm{~m}^{-2} \mathrm{~K}^{-1}$ and $83.233 \times 10^{-4} \mathrm{~W} \Omega^{-1} \mathrm{~m}^{-2} \mathrm{~K}^{-1}$, respectively. The power factor for $\mathrm{RhSb}_{3}$ at the temperatures 50, 300 and 800 $\mathrm{K}$ was $0.18076 \times 10^{-4} \mathrm{~W} \Omega^{-1} \mathrm{~m}^{-2} \mathrm{~K}^{-1}, 19.1806 \times 10^{-4} \mathrm{~W} \Omega^{-1}$ $\mathrm{m}^{-2} \mathrm{~K}^{-1}$ and $77.7349 \times 10^{-4} \mathrm{~W} \Omega^{-1} \mathrm{~m}^{-2} \mathrm{~K}^{-1}$, respectively.

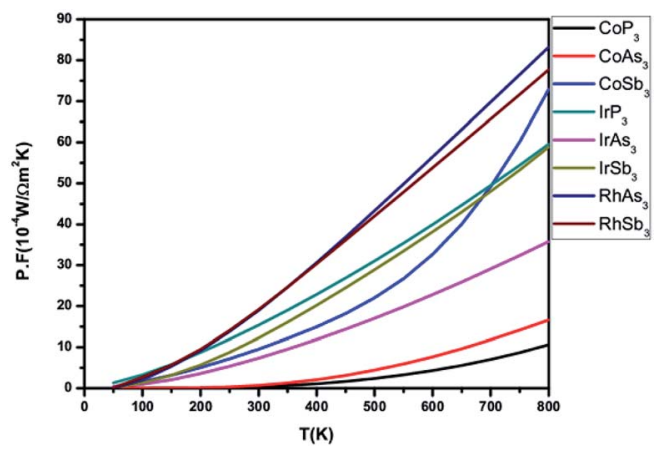

Fig. 5 Power factor at different temperatures for the binary skutterudites.

\section{Conclusions}

In conclusion, we theoretically explored the behaviour of the Seebeck coefficient, electrical conductivity, thermal conductivity and power factor of the binary semiconducting skutterdites $\left(\mathrm{CoP}_{3}, \mathrm{CoAs}_{3}, \mathrm{CoSb}_{3}, \mathrm{IrP}_{3}, \mathrm{IrAs}_{3}, \mathrm{IrSb}_{3}, \mathrm{RhAs}_{3}\right.$, and $\left.\mathrm{RhSb}_{3}\right)$, with respect to the temperature using DFT and post-DFT Boltzmann's transport theory. The Seebeck value for $\mathrm{CoSb}_{3}$ as investigated here was the highest $\left(215 \mu \mathrm{V} \mathrm{K} \mathrm{K}^{-1}\right)$ at room temperature among all the compounds under study and this result was in close agreement with the experimental findings; however, the electrical conductivity results for $\mathrm{RhAs}_{3}$ showed the highest electrical conductance of $3.736149 \times 10^{5} \Omega^{-1} \mathrm{~m}^{-1}$ among all the compounds. However, the maximum Power factor was obtained for $\mathrm{RhSb}_{3}$ with a value of $19.1806 \times 10^{-4} \mathrm{~W}$ $\Omega^{-1} \mathrm{~m}^{-2} \mathrm{~K}^{-1}$ at room temperature. We calculated the roomtemperature properties as well as the thermoelectric properties in the temperature range from 50 to $800 \mathrm{~K}$ for these compounds and compared their results.

\section{Conflicts of interest}

There are no conflicts to declare.

\section{Acknowledgements}

Muhammad Saeed acknowledges the support of start-up funding from East China University of Technology, Nanchang, JiangXi Province. We also acknowledge the financial support from the Higher Education Commission of Pakistan (HEC), project no. 20-3959/NRPU/R\&D/HEC2014/119.

\section{References}

1 G. J. Snyder and E. S. Toberer, Complex thermoelectric materials, Nat. Mater., 2008, 7, 105.

2 S. A. Yamini, T. Ikeda, A. Lalonde, Y. Pei, S. X. Dou and G. J. Snyder, Rational design of p-type thermoelectric PbTe: temperature dependent sodium solubility, J. Mater. Chem. A, 2013, 31, 8725 .

3 K. Biswas, J. He, I. D. Blum, C. IWu, T. P. Hogan, D. N. Seidman, V. P. Dravid and M. G. Kanatzidis, Highperformance bulk thermoelectrics with all-scale hierarchical architectures, Nature, 2012, 489, 414.

4 G. S. Nolas, J. Poon and M. Kanatzidis, Recent Developments in Bulk Thermoelectric Materials, MRS Bull., 2006, 31, 199.

5 G. S. Nolas, J. Sharp and H. J. Goldsmid, Thermoelectrics: Basic Principles and New Materials Developments, Springer, 2001, New York.

6 M. Yang and W. Liu, The d-p band-inversion topological insulator in bismuth-based skutterudites, Sci. Rep., 2014, 4, 5131.

7 L. Guo, X. Xu, J. R. Salvador and G. P. Meisner, Ultrafast carriers dynamics in filled-skutterudites, Appl. Phys. Lett., 2013, 102, 111905.

$8 \mathrm{~J}$. P. Fleurial, IECEC 96, Proceedings of the 31st Intersociety, 1996. 
9 B. C. Sales, D. Mandrus and R. K. Williams, Filled Skutterudite Antimonides: A New Class of Thermoelectric Materials, Science, 1996, 272, 1325.

10 X. Shi, J. Yang, J. R. Salvador, M. Chi, J. Y. Cho, H. Wang, S. Bai, J. Yang, W. Zhang and L. Chen, Multiple-Filled Skutterudites: High Thermoelectric Figure of Merit through Separately Optimizing Electrical and Thermal Transports, J. Am. Chem. Soc., 2011, 133, 7837.

11 J. M. Ziman, Electrons and Phonons: The Theory of Transport Phenomena in Solids, Oxford University Press, London, UK, 1960.

12 B. R. Nag, Electron Transport in Compound Semiconductors, Springer-Verlag, Berlin, Germany, 1980.

13 D. I. Bilc, S. D. Mahanti and M. G. Kanatzidis, Electronic transport properties of $\mathrm{PbTe}$ and $\mathrm{AgPb}_{m} \mathrm{SbTe}_{2+m}$ systems, Phys. Rev. B: Condens. Matter Mater. Phys., 2006, 74, 125202.

14 P. Villars and L. D. Calvert, Pearson's Handbook on Crystallographic Data for Intermetallic Phases, ASM International, Materials Park, Ohio, USA, 2nd edn, 1991.

15 D. J. Singh, Theoretical and computational approaches for identifying and optimizing novel thermoelectric materials, Semicond. Semimetals, 2002, 70, 125.

16 T. J. Scheidemantel, C. Ambrosch-Draxl, T. Thonhauser, J. V. Badding and J. O. Sofo, Transport coefficients from first-principles calculations, Phys. Rev. B: Condens. Matter Mater. Phys., 2003, 68, 125210.

17 G. K. H. Madsen and D. J. Singh, BoltzTraP. A code for calculating band-structure dependent quantities, Comput. Phys. Commun., 2006, 175, 67.

18 G. K. H. Madsen, Triphenylamine-Thienylenevinylene Hybrid Systems with Internal Charge Transfer as Donor Materials for Heterojunction Solar Cells, J. Am. Chem. Soc., 2006, 128, 12140.

19 L. Chaput, P. Pécheur, J. Tobola and H. Scherrer, Transport in doped skutterudites: Ab initio electronic structure calculations, Phys. Rev. B: Condens. Matter Mater. Phys, 2005, 72, 085126.

20 X. Gao, K. Uehara, D. Klug, S. Patchkovskii, J. Tse and T. Tritt, Theoretical studies on the thermopower of semiconductors and low-band-gap crystalline polymers, Phys. Rev. B: Condens. Matter Mater. Phys., 2005, 72, 125202.

21 D. Koller, F. Tran and P. Blaha, Improving the modified Becke-Johnson exchange potential, Phys. Rev. B: Condens. Matter Mater. Phys., 2012, 85, 155109.

22 B. Khan, H. A. Rahnamaye Aliabad, Saifullah, S. JalaliAsadabadi, I. Khan and I. Ahmad, Electronic band structures of binary skutterudites, J. Alloys Compd., 2015, 647, 364 .
23 M. Stoica and C. S. Lo, Electrical transport properties of Cobased skutterudites filled with $\mathrm{Ag}$ and $\mathrm{Au}$, Phys. Rev. B: Condens. Matter Mater. Phys., 2012, 86, 115211.

24 L. Chaput, P. Pécheur, J. Tobola and H. Scherrer, Transport in doped skutterudites: $\mathrm{Ab}$ initio electronic structure calculations, Phys. Rev. B: Condens. Matter Mater. Phys., 2005, 72, 085126.

25 P. Blaha, K. Schwarz, G. Madsen, D. Kvasnicka and J. Luitz, WIEN2k, An Augmented PlaneWave Plus Local Orbitals Program for Calculating Crystal Properties, WIEN2k 14.2, Vienna University of Technology, Inst. of Physical and Theoretical Chemistry, Getreidemarkt 9/156, A-1060 Vienna/Austria, 2014.

$26 \mathrm{~J}$. Ackermann and A. Wold, The preparation and characterization of the cobalt skutterudites $\mathrm{CoP}_{3}, \mathrm{CoAs}_{3}$ and $\mathrm{CoSb}_{3}$, J. Phys. Chem. Solids, 1977, 33, 1013.

27 A. Watcharapasorn, R. C. DeMattei and R. S. Feigelson, Mater. Res. Soc. Symp. Proc., 2000, 626, Z.14.1.

28 J. W. Sharp, E. C. Jones, R. K. Williams, P. M. Martin and B. C. Sales, Thermoelectric properties of $\mathrm{CoSb}_{3}$ and related alloys, J. Appl. Phys., 1995, 78, 1013.

29 C. M. Pleass and R. P. Heyding, Can. J. Chem., 1962, 40, 590.

30 G. S. Nolas, Semiconductor materials with skutterudite type crystal lattice structures optimized for selected thermoelectric properties and methods of preparation, US Pat. 6207888 B1, 2001.

31 F. Hulliger, Halbleitende Verbindungen mit SkutteruditStruktur, Helv. Phys. Acta, 1961, 34, 782.

32 T. M. Tritt, G. S. Nolas, G. A. Slack, A. C. Ehrlich, D. J. Gillespie and J. L. Cohn, Low-temperature transport properties of the filled and unfilled $\mathrm{IrSb}_{3}$ skutterudite system, J. Appl. Phys., 1996, 79, 8412.

33 G. A. Slack and V. G. Tsoukala, Some properties of semiconducting $\mathrm{IrSb}_{3}$, J. Appl. Phys., 1994, 76, 1665.

34 J. P. Odile, S. Soled, C. A. Castro and A. Wold, Crystal growth and characterization of the transition-metal phosphides copper diphosphide, nickel diphosphide, and rhodium triphosphide, Inorg. Chem., 1978, 17, 283.

35 K. T. Wojciechowski, J. Tobola and J. Leszczyiskis, Thermoelectric properties and electronic structure of $\mathrm{CoSb}_{3}$ doped with Se and Te, J. Alloys Compd., 2003, 361, 19.

36 T. Caillat, A. Borshchevsky and J.-P. Fleurial, Properties of single crystalline semiconducting $\mathrm{CoSb}_{3}$, J. Appl. Phys., 1996, 80, 4442.

37 T. Caillat, J. Fleurial and A. Borshchevsky, Progress in the search for advanced thermoelectric materials for terrestrial applications at the jet propulsion laboratory, Jet Propulsion Laboratory California Institute of Technology, 1955, p. 67. 